\title{
Using multivariate analyses for an efficient understanding of Hawaiian Islands geochemical trends
}

\author{
J. N. COOPER ${ }^{1 *}$, B. L. CLAUSEN ${ }^{1,2}$ \\ ${ }^{1}$ Loma Linda University, Dept. of Earth and Biological \\ Sciences, Loma Linda CA, 92350, USA \\ (*correspondence: jnhiday@gmail.com) \\ ${ }^{2}$ Geoscience Research Institute, 11060 Campus St., Loma \\ Linda, CA 92354, USA (bclausen@1lu.edu)
}

Geochemical analyses have traditionally been displayed in numerous bivariate or ternary plots that when used together allow for interpretation of petrologic features. These types of plots are valuable, but the need for multiple plots can be cumbersome and the geochemical complexity is unaccounted for. We demonstrate that multivariate analyses can be used to identify petrologic features more efficiently than traditional approaches.

The geochemistry of the Hawaiian Islands provides an excellent opportunity to verify the utility of multivariate analyses to identify well-established classifications, such as volcanic stage, or the Kea-Loa trend. A dataset of 450 cases from the GEOROC database was analyzed using Principal Component Analysis (PCA) and Discriminant Function Analysis (DFA).

The PCA returned four components: (1)

Compatible/incompatible elements accounted for $59 \%$ of the variance and are interpreted as extent of differentiation. (2) HREEs accounted for $13 \%$ of the variance with scores on this component related to source depth. (3) Some LILs accounted for $11 \%$ of the variance and are tentatively attributed to alkalinity and fluid effects. (4) $\mathrm{Pb}$ isotopes accounted for $5 \%$ of the variance and are related to magma reservoirs. Plots of component scores showed clear separation along the stages and trends. Two DFA were run using the stage or Kea-Loa trend as the grouping variable yielding 93.5-96\% classification accuracy.

Our results show the value of multivariate analyses for the petrologic community. A multivariate approach offers an efficient and more comprehensive tool for geochemistry research. 